\title{
Local optimal scale in a hierarchical segmentation method for satellite images
}

\section{An OBIA approach for the agricultural landscape}

\author{
Consuelo Gonzalo-Martín ${ }^{1}$ Mario Lillo-Saavedra ${ }^{2}$. \\ Ernestina Menasalvas ${ }^{1}$. David Fonseca-Luengo ${ }^{2}$. \\ Angel García-Pedrero ${ }^{1} \cdot$ Roberto Costumero $^{1}$
}

\begin{abstract}
Over recent decades, remote sensing has emerged as an effective tool for improving agriculture productivity. In particular, many works have dealt with the problem of identifying characteristics or phenomena of crops and orchards on different scales using remote sensed images. Since the natural processes are scale dependent and most of them are hierarchically structured, the determination of optimal study scales is mandatory in understanding these processes and their interactions. The concept of multi-scale/multiresolution inherent to OBIA methodologies allows the scale problem to be dealt with. But for that multi-scale and hierarchical segmentation algorithms are required. The question that remains unsolved is to determine the suitable scale segmentation that allows different objects and phenomena to be characterized in a single image. In this work, an adaptation of the Simple Linear Iterative Clustering (SLIC) algorithm to perform a multi-scale hierarchical segmentation of satellite images is proposed. The selection of the optimal multi-scale segmentation for different regions of the image is carried out by evaluating the intravariability and inter-heterogeneity of the regions obtained on each scale with respect to the parent-regions defined by the coarsest scale. To achieve this goal, an objective function, that combines weighted variance and the global Moran index, has been used. Two different kinds of experiment have been carried out, generating the number of regions on each scale through linear and dyadic approaches. This methodology has allowed, on the one hand, the detection of objects on different scales and, on the other hand, to represent them all in a single image. Altogether, the procedure provides the user with a better comprehension of the land cover, the objects on it and the phenomena occurring.
\end{abstract}

This work has been funded by the Centro de Recursos Hídricos para la Agricultura y la Minería (CONICYT/FONDAP/1513001).

Consuelo Gonzalo-Martín

consuelo.gonzalo@upm.es

1 Centro de Tecnología Biomédica, Universidad Politécnica de Madrid, Campus Montegancedo, 28223 Pozuelo de Alarcón, Madrid, Spain

2 Faculty of Agricultural Engineering, Universidad de Concepción, Vicente Méndez 595, Chillán, Chile 


\section{Introduction}

Over recent decades, remote sensing has emerged as an effective tool to improve agriculture productivity (Ozdogan et al. 2010). In particular, many works have dealt with the problem of identifying characteristics or phenomena of crops and orchards on different scales using remote sensed images (Vieira et al. 2012; Duveiller and Defourny 2010). This is a complex task that needs to take different factors into account, such as those related to agronomy, scale of study, image resolution (Peña-Barragan et al. 2011), some of which have a high degree of dependence between them.

Woodcock and Strahler (1987) expounded the importance of the factor of scale in the identification of objects present in remote sensing imagery. Understanding the concept of scale of an object as the level of aggregation and abstraction at which this object can be described (Benz et al. 2004). In this sense, the identification of objects in remote sensing imagery depends greatly on the relationship between the spatial resolution of the images and the size of the objects. Thus, the bigger the object is with respect to the image spatial resolution, the more likely it is to be fragmented. On the other hand, if the size of the object is very small in relation to the spatial resolution it could happen that the object is not identified. Therefore, the availability of high resolution satellite images is essential to allow small objects to be identified on fine scales. However, although it is a fact that satellite images with high spatial resolution exhibit high levels of detail; traditional image processing algorithms based on single pixel analysis are often not capable of extracting the entire information; this situation potentially leads to lower accuracy in the traditional remote sensing tasks (classification, change detection, among others) (Mynt et al. 2011). In recent years, object-based image analysis methodologies (OBIA) have become popular in high spatial resolution image processing and they have proven to be an alternative to the pixel-based image analysis. Different publications suggest that better results can be expected (Blaschke 2010).

The images in the OBIA paradigm are interpreted as a set of areas in the scene (segments) with homogeneous characteristics, which are considered as the minimal information unit instead of the pixels (Benz et al. 2004). The main advantages of the OBIA approach are:

- Reduced sensitivity to noise. Processing semantic objects rather than individual pixels or meaningless objects can help to overcome some of own artifacts of high resolution image processing.

- Use of features of different nature. Different features can be used to characterize the objects (shape, size, texture and contextual relationships). This last aspect allows high level rules to be defined.

- Reduced computational requirements (Homeyer et al. 2010). 
Even though in recent years most of the OBIA approaches have been developed in the area of Remote Sensing and Geographical Information Systems (Blaschke 2010; Hay and Castilla 2008), applications in other areas like medicine (Chitiboi et al. 2013) or neurosciences (Lucchi et al. 2012) can also be found.

The two main steps in the OBIA approach are an initial segmentation followed by a classification process (Baatz et al. 2008). The segmentation generates segments by grouping neighboring pixels according to certain homogeneity criteria, such as spectral characteristics, texture, context or spatial relationships. In the classification process, each segment is assigned to a class depending on its features (Vieira et al. 2012). Numerous researches have shown that the segmentation process is a critical step in the OBIA approach. This is due to the fact that the results of the classification step strongly depend on the initial segmentation (Gao and Mas 2008; Yan et al. 2006).

Furthermore the accepted idea is that natural processes are scale dependent, it is also known that most of them are hierarchically structured. Therefore an appropriate understanding of these processes and interactions between them requires the determination of optimal study scales (Woodcock and Strahler 1987; Levin 1992; Paul and Philip 2000) and the spatial relationships between the regions at different scales (Burnett and Blaschke 2003). These aspects are especially interesting for the identification, characterization and analysis of crops, as their inherent complexity makes multi-scale analysis a requirement in dealing with the different sizes of the interest objects in the image (Baatz and Schäpe 2000; Duveiller and Defourny 2010).

Some studies have designed methodologies for estimating a single optimal scale segmentation for the whole scene (Espindola et al. 2006; Kim et al. 2009). Though other studies have implemented a multi-scale approach (Zhou and Troy 2009; Trias-Sanz et al. 2008) to address this problem, in these cases the selection of the segmentation scales is typically based on an extensive knowledge of the study site (Johnson and Xie 2011). Therefore, as far as our knowledge is concerned, the unsupervised definition of the most suitable scale for each land cover in a scene remains unsolved.

The concept of multi-scale/multi-resolution inherent to OBIA methodologies allows the scale problem to be dealt with (Zhong et al. 2005; Burnett and Blaschke 2003), by defining a hierarchy of objects at different scales through hierarchical segmentation algorithms.

The introduction of multi-resolution/hierarchical segmentation using the Fractal Net Evaluation (FNEA) approach by in Definiens Professional Version 5.0 (Baatz and Schäpe $2000)$ was a milestone in research into multi-resolution segmentation for remote sensing imagery. The implemented multiresolution segmentation technique uses homogeneity criteria based on color and shape, and a scale parameter in combination with local and global optimization techniques. The tuning of different parameters requires an expert knowledge, not always available; moreover, this task usually requieres a lot of time. A fuzzy approach which automatically selects the parameters of the segmentation used in multi-resolution approach was proposed by (Maxwell and Zhang 2005). In any case, the license cost of this software prevents its use in many applications. Since then, other multiresolution/hierarchical segmentation techniques have been proposed in the literature (Chen et al. 2003; Zhong et al. 2005; Li et al. 2008).

In recent years, new types of image segmentation methods, known as superpixel (SP) methods (Achanta et al. 2012), have been developed in the area of computer vision. A SP is a small, local, and coherent cluster that contains a statistically homogeneous image region according to certain criteria such as color, texture, etc. (Ren and Malik 2003). Superpixels reduce the influence of noise, preserve most edges of images, are approximately uniform in size and shape, and improve the computational speed of next processing steps. 
Many approaches to generating SP can be found in the literature, most of them are suited to a particular application. For example, the method proposed in (Felzenszwalb and Huttenlocher 2004) provides a very good adherence to image boundaries, but the generated superpixels are large and very irregular, which produce a poor segmentation performance and under-segmentation error. In Achanta et al. (2012) an empirical comparison of five state-of-the-art algorithms were carried out. The study compares the boundary adherence of the superpixels, segmentation speed, and performance when used as a preprocessing step in a segmentation framework. As result of this study the authors concluded that the SLIC algorithm has a better overall performance than the other SP algorithms. However, SLIC has a drawback: the segmentation on different scales does not have a hierarchical structure like for example QuickShift (Vedaldi and Soatto 2008).

This work provides two main contributions:

1. An adapted multi-scale hierarchical segmentation algorithm to deal with objects that are scale dependent and hierarchically related in a satellite scene.

2. A method for selecting the local optimal scale associated to different regions in the images. The selection of the optimal multi-scale segmentation is carried out by evaluating the intra-variability and inter-heterogeneity of the regions defined by the coarsest scale. This methodology is based on the evaluation method presented in (Johnson and Xie 2011), where the complete scene is used for evaluation, but adapting the one to deal with the hierarchical image segmentation process.

The methodology has been applied to a scene corresponding to an agricultural area, where different kinds of land covers, showing a high variability, are represented.

\section{Materials and methods}

\subsection{Data set}

To test the methodology proposed in this work a scene of an agricultural area with a high local variability has been selected. The study area is located in the central irrigated valley of Chile. It corresponds to a rotation sugar beet crop of Sandrina, Magnolia and Donella varieties. The plantation frame is $0.5 \mathrm{~m}$ between rows and $0.07 \mathrm{~m}$ between plants, with a center pivot irrigation system. The total area of the farm is around $450 \mathrm{ha}$, and the area of crop covered for the center pivot (study site) is around 17 ha. The corresponding multispectral satellite image was collected on January 18 th, 2013 by the Pléiades Satellite. ${ }^{1}$ The coordinates of the center of the scene are: $36^{\circ} 31^{\prime} 54^{\prime \prime} \mathrm{S} ; 72^{\circ} 08^{\prime} 03^{\prime \prime} \mathrm{O}$ ). The area the scene is 420 hectares and corresponds to $1024 \times 1024$ pixels. The collected image has a spatial resolution of 2 meters and corresponds to ORTHO-BASIC products, characterized by a basic radiometric normalization (for the calibration of the detector) and geometric correction (WGS84/UTM 19S projection). The spectral and spatial resolutions are presented in Table 1:

${ }^{1}$ http://www.landinfo.coni/pleiades-satellite-imagery.htm 
Table 1 Spectral and spatial characteristics of the multispectral image taken by the Pléiades satellite

\begin{tabular}{lll}
\hline Band Name & Band Width $(\mathrm{nm})$ & Spatial Resolution $(\mathrm{m})$ \\
\hline Blue & $430-550$ & 2 \\
Green & $490-610$ & 2 \\
Red & $600-720$ & 2 \\
Near Infrared & $750-950$ & 2 \\
\hline
\end{tabular}

A color composition of the described multispectral scene is shown in Fig. 1.

\subsection{Superpixels algorithm: SLIC}

The multi-scale hierarchical segmentation algorithm proposed in this work is based on a modified version of the SLIC algorithm (Garcia-Pedrero et al. 2015). The original SLIC algorithm (Achanta et al. 2010) is based on the well-known k-means method to group pixels of RGB/CIELab images into superpixels. The CIELab color space is an international standard for color measurements, adopted by the Commision Internationale d'Eclairage (CIE) in 1976.

SLIC superpixels are generated according to two criteria: spectral similarity (limited to three channels) and spatial proximity. A detailed description of the original SLIC algorithm working in CIELab color space is given by Achanta et al. (2010).

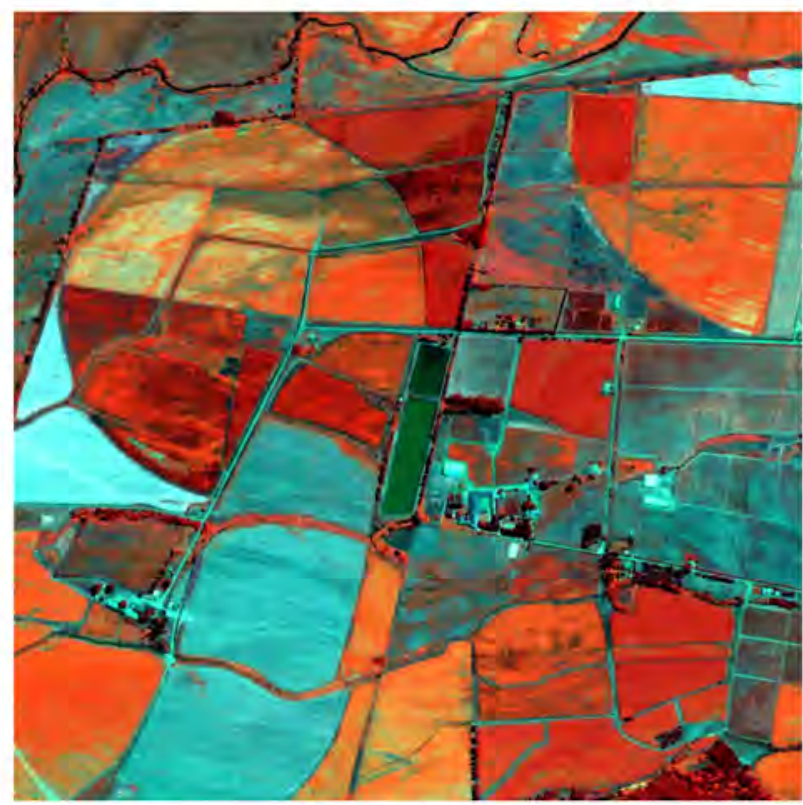

Fig. 1 Color composition (Near Infrared-Green-Blue) of the analyzed scene. The satellite image was collected on January 18th, 2013 by Pléiades Satellite, in the central irrigated valley of Chile $\left(36^{\circ} 31^{\prime} 54^{\prime \prime} \mathrm{S} ; 72^{\circ}\right.$ $\left.08^{\prime} 03^{\prime \prime} \mathrm{O}\right)$ 
In the SLIC procedure, the generation of superpixels is based on the assumption that limiting the search space to a region proportional to the desired superpixel size reduces considerably the calculation time. In fact, its computational complexity is linear in the number of pixels in the image (Achanta et al. 2012). Moreover, a weighted distance that combines spectral and spatial proximity allows controlling the size and compactness of the superpixel (Achanta et al. 2012). Therefore, it has two parameters: $k$, the desired number of superpixels, and $c$, the compactness factor. A larger value of $c$ emphasizes the importance of the spatial proximity resulting in more compact superpixels.

The modified version of SLIC extends the definition of spectral proximity provided by SLIC to work with multispectral images of $B$ bands. The first step of the segmentation framework begins with the sampling of $k$ initial cluster centers on a regularly spaced grid of $g$ pixels. The initial centers are defined as:

$$
C_{i}=\left[p^{1}, p^{2}, \ldots, p^{B}, l_{x}, l_{y}\right]^{T}, \quad i=1,2, \ldots, k
$$

where $p^{b}$ represents the spectral value in band $b-t h$ of pixel $p$ at position $l_{x}$ and $l_{y}$, and $B$ denotes the number of spectral bands. To produce similar sized superpixels, the grid interval is defined as $g=N / k$, where $N$ is the total number of pixels in the image. $g$ determines the size of the superpixels, the greater value of $g$, the larger the superpixels.

In the next step, each pixel $p$ is associated to the nearest cluster center $C_{i}$ whose search space overlaps its location. The search region is enclosed to an area of $2 g \times 2 g$ pixels around each superpixel center. Then the cluster centers are updated to be the mean vector of all pixels belonging to the cluster. Both steps are repeated iteratively until a maximum of 10 iterations, since no further significant changes in superpixel quality could be observed (Achanta et al. 2010).

The clustering distance is a weighted relationship between spectral and spatial measures. The first measure ensures superpixel homogeneity, and the second one enforces compactness and regularity in superpixels shape. In order to work with multispectral images, the spectral square distance between pixels $i$ and $j$ is defined as follows:

$$
d_{c}^{2}=\sum_{b=1}^{B}\left(p_{i}^{b}-p_{j}^{b}\right)^{2}
$$

The spatial square distance is calculated as:

$$
d_{s}^{2}=\left(l_{x}^{i}-l_{x}^{j}\right)^{2}+\left(l_{y}^{i}-l_{y}^{j}\right)^{2}
$$

where $l_{x}^{i}$ and $l_{y}^{i}$ denote the position of the pixel $i^{t_{h}}$.

Finally, the clustering distance is calculated as:

$$
D=d_{c}+\left(\frac{c}{g}\right) d_{s}
$$

where $c$ controls the compactness of the superpixels. Preliminary results suggest that a $3.9 \%$ of the maximum pixel value in image as $c$ is optimal.

\subsection{Methodology}

The methodology we propose is made up of two main processing modules: 1) image segmentation, and ii) segmentation evaluation. They are detailed below; 


\subsubsection{Hierarchical image segmentation}

Even though superpixels present some good properties mentioned before, it is not able to generate a hierarchical segmentation. Our approach fills this gap by proposing an adaptation to SLIC that makes the generation of a hierarchical multi-scale segmentation possible.

Based on the fact that in SLIC, low values of $k$ results in a coarser segmentation in comparison to finer segmentations obtained increasing the values of $k$, we propose to generate segmentations at different scales by varying the size of the superpixels and then combining the resulting segmentations.

To establish the different segmentation scales, a vector $K=\left[k_{1}, k_{2}, \ldots, k_{n}\right]$ containing different values for the size of the superpixels in ascending order is defined. Each value of the vector corresponds to a different scale (i). Consequently, segments corresponding to a segmentation carried out according to $k_{i}$ will be larger than those of the segmentation according to $k_{i+1}$. A segment $A^{i}$ in scale $i$ is considered as the parent of a segment $B^{i+1}$ in scale $i+1$, if the area of $B^{i+1}$ can be entirely covered by segment $A^{i}$. The parent-children relationship between segments is shown in Fig. 2 . In order to ensure that the resulting segmentation process is hierarchical, segments on scale $k_{i+1}$ are partitioned by segments on scale $k_{i}$, obtaining a new segmentation that generally contains a higher number of segments than in the segmentation achieved in scale $k_{i+1}$. To match the number of superpixels in scale $i+1$ to its original number of superpixels, smaller size segment are joined to the most similar neighboring segment belonging to the same parent. We use euclidean distance in the spectral space (color) to define the similarity between neighboring segments.

\subsubsection{Determination of the optimal local scale}

One important contribution of our methodology is that it provides different optimal scales for different image regions, against the results shown in (Johnson and Xie 2011), where an optimal scale for the whole image is yielded. This fact is especially relevant in images where the inter-region variability is high, as it is the case of some crops in satellite images.

A general overview of the scheme proposed is shown in Fig. 3.

In order to determine the optimal scale for each image region generated by the hierarchical segmentation process, the following inter and intra-segment quality measures are used:

- the global Moran index is used to measure the inter-heterogeneity between segments;

- the weighted variance is used for intra-segment homogeneity.

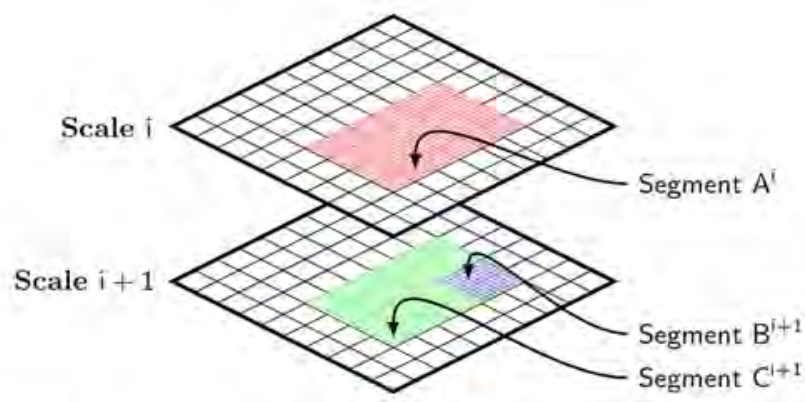

Fig. 2 Segment $A^{i}$ is considered the parent of segments $B^{i+1}$ and $C^{i+1}$, as both segments can be covered entirely by segment $A^{i}$. Each square of the grid represents an image pixel 


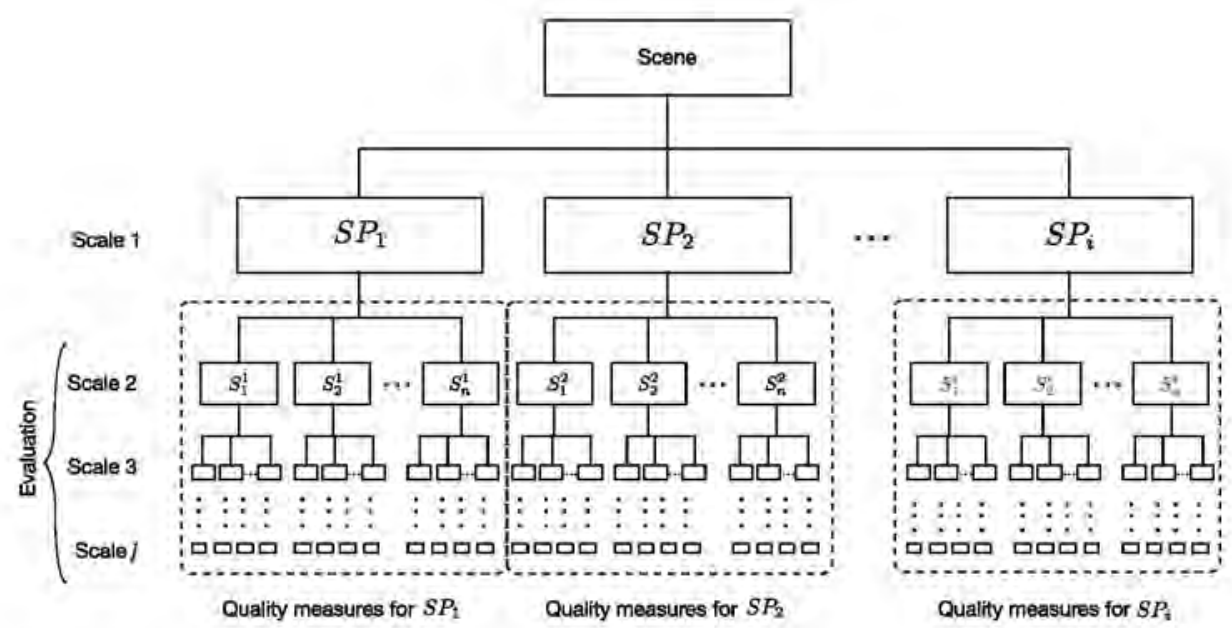

Fig. 3 General overview of the evaluation process

The global Moran index measures the correlation between segments. It is used to evaluate the similarity of a segment with its neigbborhood and is calculated as follows:

$$
M I_{i}^{p}=\frac{n \sum_{u=1}^{n} \sum_{\nu=1}^{n} w_{u v}\left(y_{u}^{i}-\hat{y}^{p}\right)\left(y_{v}^{i}-\hat{y}^{p}\right)}{\sum_{u=1}^{n}\left(y_{u}^{p}-\hat{y}^{p}\right)^{2}\left(\sum_{u \neq v} \sum w_{u v}\right)}
$$

where:

- $n$ is the total number of regions on the analysis scale that are related to the parent segment $p$.

- $\quad w$ is an adjacency matrix among neighboring regions that are relatives of the segment $p$ on scale 1 (parent), therefore $w_{u v}$ is equal to 1 if regions $u$ and $v$ are adjacent, otherwise, $w_{t i v}=0$.

- $\quad y_{u}^{i}$ is the spectral average of a particular region $(u)$ on seale $i$.

- $\hat{y}^{p}$ is the spectral average of a parent.

In order to calculate the similarity on a particular scale level only those regions belonging to a certain parent segment $p$ on scale 1 are considered.

The Global Moran index ranges from the value -1 , that corresponds to the perfect dispersion, to 1 , that indicates a complete correlation. The 0 value corresponds to a random correlation.

The weighted variance is used for intra-segment quality measurement, and is calculated as follows:

$$
w \operatorname{Var}^{i}=\frac{\sum_{u=1}^{n} a_{u}^{i} v_{u}^{i}}{\sum_{u=1}^{n} a_{u}^{i}}
$$

where:

- $\quad v_{u}^{i}$ is the variance of the pixels that compounds the segment $u$ on scale $i$.

- $a_{u}^{i}$ corresponds to the size of segment $u$ in scale $i$.

- $n$ is the total number of regions on the analysis scale that are related to the parent segment $p$. 
Values of variance close to zero indicate homogeneity of a segment. Note that, when calculating this measurement, larger sized segments have a greater effect on the evaluation than those of a smaller size. In this paper, the best scale is defined as the value of the objective function $\left(F^{i}\right)$ closer to zero, $F^{i}$ being ealculated by adding the normalized (range (0-1) of intra and inter-segments measurements.

The objective function (OF) is calculated for each scale $i$ as:

$$
F^{i}=w \operatorname{Var}^{i}+M I^{i}
$$

where:

- $F^{i}$ represents $\mathrm{OF}$, calculated using the parent segment $(p)$, at the scale $1^{\text {st }}$, and the each related segments at scale $i$.

\subsubsection{Experiments}

Two different experiments were carried out. In the first one, the number of superpixels associated with each scale was generated with a linear spacing. In the second one, the number of superpixels on each scale was generated with a dyadic spacing $\left(2^{n}\right)$. The number of scales used in both experiments was 7, varying from 64 to 4,096 superpixels. Table 2 details the number of superpixels for each scale and for each approach. It can be noted that except for the first and the $7^{\text {th }}$ scale, the number of superpixels is always larger for the linear approach than for the dyadic one.

\section{Results and discussion}

In this work the four spectral bands (Blue, Green, Red and Near Infrared) of the multispectral image (Fig. 1) were used in the segmentation process and in the calculation of the metrics to determine the optimal scale for each parent superpixel.

The results are detailed in Fig. 4. The first column corresponds to the results obtained with the linear approach, while the second row shows the results obtained with the dyadic approach. The optimal scales provided for the hierarchical segmentation algorithm for each parent superpixel on the $1^{s t}$ scale are represented in the first row (Fig. $4 \mathrm{a}$ and b. Figure $4 \mathrm{c}$ and $\mathrm{d}$ represent the spatially distributed optimal scale values (from 1 to 7 ). Figure $4 \mathrm{e}$ and $\mathrm{f}$ display the edge of the segments for all scales.

As it has been mentioned before the number of superpixels is always larger for the linear approach than for the dyadic one for each particular scale. Thus it can be seen in Fig. 4a and $\mathrm{b}$ that in the linear approach most of the parent superpixels have assigned optimal scales from 4th to 7th, while coarser scales are assigned in the linear one. The higher number of superpixels on the intermediate scale for the linear approach (see Table 2) produces an abrupt change from coarsest to finest scale, giving rise to an over-segmentation in some

Table 2 K-vectors values for linear and dyadic approach

\begin{tabular}{|c|c|c|c|c|c|c|c|}
\hline \multicolumn{8}{|l|}{ Scale } \\
\hline Approach & 1 & 2 & 3 & 4 & 5 & 6 & 7 \\
\hline Linear & 64 & 736 & 1048 & 2080 & 2752 & 3424 & 4096 \\
\hline Dyadic & 64 & 128 & 256 & 512 & 1024 & 2048 & 4096 \\
\hline
\end{tabular}




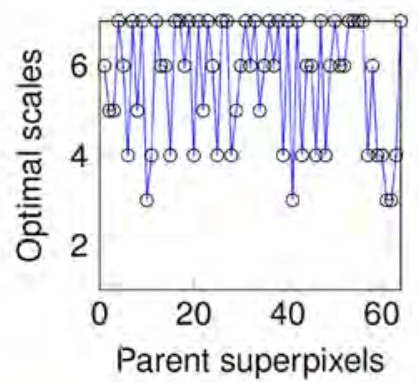

4.1

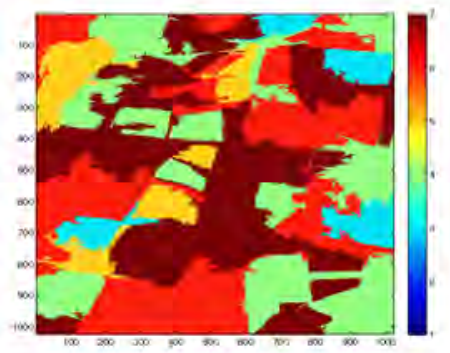

4.3

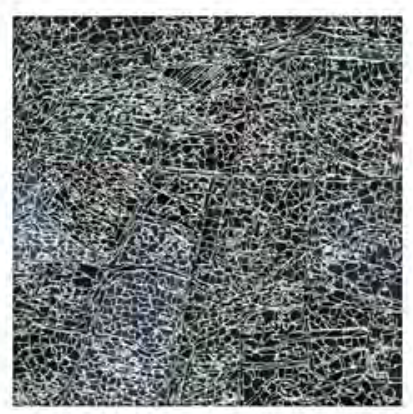

4.5

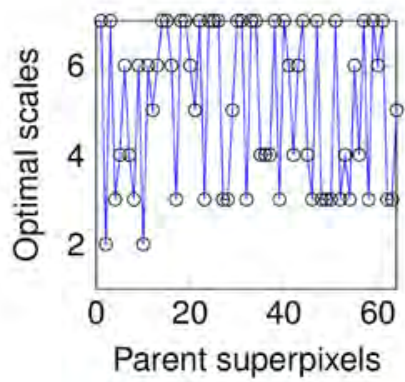

4.2

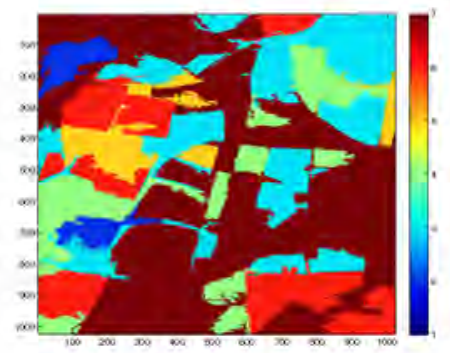

4.4

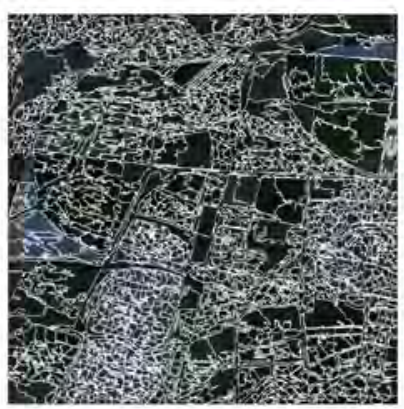

4.6

Fig. 4 First column: linear approach. Second column: dyadic approach, a and b Optimal scales for each parent superpixel, $\mathbf{c}$ and $\mathbf{d}$ Optimal scale spatially distributed. $\mathbf{e}$ and $\mathbf{f}$ Edge of segments

homogeneous areas, as it can be appreciated in Fig. 4c, d, e and f. It can be observed that there are different well-recognized structures in Fig. 4d but not in Fig. 4c, such as: plots, roads and irrigation circular areas, known as pivots. This shows that the dyadic approach provides more realistic local optical scales than the linear one. In addition, in Fig. $4 \mathrm{~d}$ it is clear that different scales are associated to different regions. Figure 5 shows the final segmentation result for the experiment in which the scales were generated through dyadic spacing. 


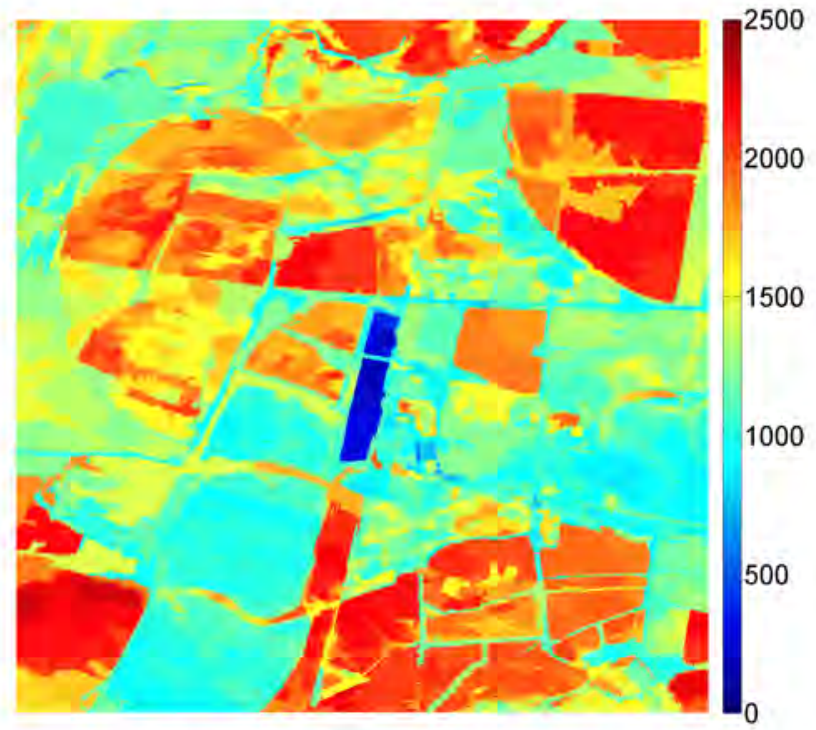

Fig. 5 Segmented image through the dyadic approach

This image has been obtained from the Infrared band by assigning to each segment the average digital value of the pixels included in it.

It can be observed that agricultural management units with high variability in the original scene has been identified as homogeneous areas, it is very important for generating information to improve the agricultural management.

\section{Conclusions}

The two main contributions of this work are: i) an adapted multi-scale hierarchical segmentation algorithm for satellite images based on the Simple Linear Iterative Clustering (SLIC) algorithm and ii) a method to select the local optimal scale associated to different regions in the images. The determination of the local optimal scales is carried out by evaluating the intra-variability and inter-heterogeneity of the regions obtained on each scale in respect to the parents-regions defined by the coarsest scale. An objective function that combines weighted variance and the global Moran index, was used to select the optimal scale. The number of regions on each scale has been determined in two different ways: linearly and dyadically spacing. From the results it can conclude that the method proposed allows users to benefit from detecting objects in an image at different scales. This provides the user with a better comprehension of the land-cover, its objects and phenomena. This fact is especially relevant in images where the inter-region variability is high, as is the case of natural land cover in satellite images. It should be noted that the segmentation results are highly dependent on the approach used to determine the scales. The dyadic approach provides more realistic local optical scales, in the sense that, in the segmentation provided by this approach, different structures (plots, roads, and pivots) can be well recognized but not 
in the segmentation based on the linear approach, due to the over-segmentation produced in this case.

Acknowledgments A. García-Pedrero (grant 216146) and D. Fonseca-Luengo acknowledge the support for the realization of their doctoral thesis to the Mexican National Council of Science and Technology (CONACyT) and the National Commission for Scientific and Technological Research (CONICYT), respectively.

\section{References}

Achanta, R., Shaji, A., Smith, K., Lucchi, A., Fua, P., \& Süsstrunk, S. (2010). Slic superpixels. École Polytechnique Fédéral de Lausssanne (EPFL), Tech Rep 149300.

Achanta, R., Shaji, A., Smith, K., Lucchi, A., Fua, P., \& Süsstrunk, S. (2012). SLIC superpixels compared to state-of-the-art superpixel methods. IEEE Transactions on Pattern Analysis and Machine Intelligence, 34(11), 2274-2282.

Baatz, M., \& Schäpe, A. (2000). Multiresolution segmentation: an optimization approach for high quality multi-scale image segmentation. Angewandte Geographische Informationsverarbeitung XII (pp. 12-23).

Baatz, M., Hoffmann, C., \& Willhauck, G. (2008). Progressing from object-based to object-oriented image analysis. In T. Blaschke, S. Lang \& G. Hay (Eds.), Object-based image analysis, lecture notes in geoinformation and cartography (pp. 29-42). Berlin: Springer.

Benz, U.C., Hofmann, P., Willhauck, G., Lingenfelder, I., \& Heynen, M. (2004). Multi-resolution, object-oriented fuzzy analysis of remote sensing data for gis-ready information. ISPRS Joumal of Photogrammetry and Remote Sensing, 58(3), 239-258.

Blaschke, T. (2010). Object based image analysis for remote sensing. ISPRS Journal of Photogrammetry and Remote Sensing, 65(1), 2-16.

Burnett, C., \& Blaschke, T. (2003). A multi-scale segmentation/object relationship modelling methodology for landscape analysis. Ecological Modelling, 168(3), 233-249.

Chen, Q.X., Luo, J.C., Zhou, C.H., \& Pei, T. (2003). A hybrid multi-scale segmentation approach for remotely sensed imagery. In Proceedings of IEEE International Geoscience and Remote Sensing Symposium (IGARSS) (Vol. 6, pp. 3416-3419).

Chitiboi, T., Hennemuth, A., Tautz, L., Stolzmann, P., Donati, O.F., Linsen, L., \& Hahn, H.K. (2013). Automatic detection of myocardial perfusion defects using object-based myocardium segmentation. In Computing in Cardiology Conference (CinC) (pp. 639-642).

Duveiller, G., \& Defourny, P. (2010). A conceptual framework to define the spatial resolution requirements for agricultural monitoring using remote sensing. Remote Sensing of Environment, 114(11), 2637-2650.

Espindola, G.M., Camara, G., Reis, I.A., Bins, L.S., \& Monteiro, A.M. (2006). Parameter selection for region-growing image segmentation algorithms using spatial autocorrelation. International Joumal of Remote Sensing, 27(14), 3035-3040.

Felzenszwalb, P.F., \& Huttenlocher, D.P. (2004). Efficient graph-based image segmentation. International Journal of Computer Vision, 59(2), 167-181.

Gao, Y., \& Mas, J. (2008). A comparison of the performance of pixel-based and object-based classifications over images with various spatial resolutions. Proceedings of GEOBIA 2008 Pixels, Objects, Intelligence: Geographic Object-Based Image Analysis for the 21st Century, 2(1), 27-35.

Garcia-Pedrero, A., Gonzalo-Martin, C., Fonseca-Luengo, D., \& Lillo-Saavedra, M. (2015). A GEOBIA methodology for fragmented agricultural landscapes. Remote Sensing, 7(1), 767-787.

Hay, G., \& Castilla, G. (2008). Geographic object-based image analysis (geobia): A new name for a new discipline. In T. Blaschke, S. Lang \& G. Hay (Eds.), Object-based image analysis, lecture notes in geoinformation and cartography (pp. 75-89). Berlin: Springer.

Homeyer, A., Schwier, M., \& Hahn, H.K. (2010). A generic concept for object-based image analysis. In VISAPP (pp. 530-533).

Johnson, B., \& Xie, Z. (2011). Unsupervised image segmentation evaluation and refinement using a multiscale approach. ISPRS Joumal of Photogrammetry and Remote Sensing, 66(4), 473-483.

Kim, M., Madden, M., Warner, T.A., et al. (2009). Forest type mapping using object-specific texture measures from multispectral ikonos imagery: segmentation quality and image classification issues. Photogrammetric Engineering and Remote Sensing, 75(7), 819-829.

Levin, S. (1992). The problem of pattern and scale in ecology: the Robert H. MacArthur award lecture. Ecology, 73(6), 1943-1967. 
Li, H., Gu, H., Han, Y., \& Yang, J. (2008). An efficient multi-scale segmentation for high-resolution remote sensing imagery based on statistical region merging and minimum heterogeneity rule. In Earth Observation and Remote Sensing Applications, International Workshop on EORSA (pp. 1-6). IEEE.

Lucchi, A., Smith, K., Achanta, R., Knott, G., \& Fua, P. (2012). Supervoxel-based segmentation of mitochondria in EM image stacks with learned shape features. IEEE Transactions on Medical Imaging, 31(2), $474-486$.

Maxwell, T., \& Zhang, Y. (2005). A fuzzy logic approach to optimization of segmentation of object-oriented classification. In Proceedings of SPIE 50th annual meeting - optics \& photonics (Vol. 5909, pp. 1-11).

Mynt, S.W., Gobera, P., Brazela, A., Grossman-Clarke, S., \& Weng, Q. (2011). Per-pixels vs object-based classif ication of urban land cover extraction using high spatial resolution imagery. Remote Sensing of Environment, 115(5), 1145-1161.

Ozdogan, M., Yang, Y., Allez, G., \& Cervantes, C. (2010). Remote sensing of irrigated agriculture: opportunities and challenges. Remote Sensing, 2(9), 2274-2304.

Paul, T., \& Philip, H. (2000). High spatial resolution remote sensing data for forest ecosystem classification: an examination of spatial scale. Remote Sensing of Environment, 72(3), 268-289.

Peña-Barragan, J., Ngugi, M., Plant, R., \& Six, J. (2011). Object-based crop identification using multiple vegetation indices, textural features and crop phenology. Remote Sensing of Environment, 115(6), 13011316.

Ren, X., \& Malik, J. (2003). Learning a classification model for segmentation. In Computer vision, 2003. ninth IEEE international conference on proceedings (Vol. 1, pp. 10-17). IEEE.

Trias-Sanz, R., Stamon, G., \& Louchet, J. (2008). Using colour, texture, and hierarchial segmentation for high-resolution remote sensing. ISPRS Journal of Photogrammetry and Remote Sensing, 63(2), 156168.

Vedaldi, A., \& Soatto, S. (2008). Quick shift and kernel methods for mode seeking. In Computer visionECCV 2008 (pp. 705-718). Springer.

Vieira, M., Formaggio, A., Rennó, C., Atzberger, C., Aguiar, D., \& Mello, M. (2012). Object based image analysis and data mining applied to a remotely sensed landsat time-series to map sugarcane over large areas. Remote Sensing of Environment, 123, 553-562.

Woodcock, C.E., \& Strahler, A.H. (1987). The factor of scale in remote sensing. Remote Sensing of Ervironment, 21(3), 311-332.

Yan, G., Mas, J., Maathuis, B., Xiangmin, Z., \& Van Dijk, P. (2006). Comparison of pixel-based and objectoriented image classification approachesa case study in a coal fire area, Wuda, Inner Mongolia, China. International Joumal of Remote Sensing, 27(18), 4039-4055.

Zhong, C., Zhongmin, Z., DongMei, Y., \& Renxi, C. (2005). Multi-scale segmentation of the high resolution remote sensing image. In Proccedings of IEEE Intemational Geoscience and Remote Sensing Symposium (IGARSS) (pp. 3682-3684).

Zhou, W., \& Troy, A. (2009). Development of an object-based framework for classifying and inventorying human-dominated forest ecosystems. International Joumal of Remote Sensing, 30(23), 6343-6360. 